\section{Serum klotho concentrations inversely correlate with the severity of nailfold capillaroscopic patterns in patients with systemic sclerosis}

\author{
R. Talotta ${ }^{1}$, F. Rigamonti ${ }^{1}$, T. Letizia ${ }^{2}$, S. Bongiovanni ${ }^{1}$, M.C. Ditto', \\ M. Antivalle ${ }^{1}$, S. Santandrea ${ }^{1}$, F. Atzeni ${ }^{3}$, T. Vago ${ }^{2}$, P. Sarzi-Puttini ${ }^{1}$ \\ ${ }^{1}$ Department of Rheumatology, University Hospital ASST-FBF-Sacco, Milano, Italy; \\ ${ }^{2}$ Endocrinology and Rheumatology Laboratory, University Hospital ASST-FBF-Sacco, Milano, Italy; \\ ${ }^{3}$ Rheumatology Unit, University of Messina, Messina, Italy
}

\section{SUMMARY}

Klotho is a transmembrane and soluble glycoprotein that governs vascular integrity. Previous studies have demonstrated reduced serum klotho concentrations in patients with systemic sclerosis ( $\mathrm{SSc}$ ), and it is known that klotho deficiency can impair the healing of digital ulcers related to microvessel damage. The aim of this study was to evaluate the association between serum klotho levels and nailfold capillaroscopic abnormalities in SSc patients. We retrospectively enrolled 54 consecutive patients with SSc diagnosed on the basis of the 2013 EULAR/ACR criteria [11 with diffuse SSc; 47 females; median age 68.0 years (IQ 18); median disease duration 11.0 years (IQ 7)]. Serum klotho concentrations were determined by means of an enzyme-linked immunosorbent assay. On the basis of the 2000 classification of Cutolo et al., 14 patients had normal nailfold capillaroscopic findings, 8 had an early scleroderma pattern, 21 an active scleroderma pattern, and 11 a late scleroderma pattern. The median serum klotho concentration was $0.29 \mathrm{ng} / \mathrm{mL}$ (IQ 1). Regression analysis of variation showed an inverse correlation between serum klotho concentrations and the severity of the capillaroscopic pattern $(\mathrm{p}=0.02 ; \mathrm{t}-2.2284)$, which was not influenced by concomitant treatment. Logistic regression did not reveal any significant association between the risk of developing digital ulcers and nailfold capillaroscopic patterns, serum klotho levels, or concomitant medications. The presence of avascular areas significantly correlated with calcinosis $(\mathrm{p}=0.006)$. In line with previous studies, our findings confirm that klotho plays a role in preventing microvascular damage detected with nailfold capillaroscopy.

Key words: Klotho; Systemic sclerosis; Nailfold videocapillaroscopy; Biomarkers.

\section{INTRODUCTION}

ystemic sclerosis ( $\mathrm{SSc}$ ) is a connective tissue disease that mainly affects middle-aged women and is clinically characterized by a wide range of manifestations, including Raynaud's phenomenon, skin and visceral fibrosis, digital ulcers (DUs), subcutaneous calcinosis and pulmonary artery hypertension (PAH). Unlike that of other connective tissue diseases, the pathogenesis of SSc is mainly related to endothelial dysfunction and impaired reparative processes, with the immune system playing a secondary role (1).

Nailfold capillaroscopy is an instrumental procedure that can detect signs of mi- crovessel damage in the early phases of the disease (2). An altered (scleroderma) capillaroscopic pattern therefore has diagnostic and prognostic value, and has now been included in the SSc classification criteria $(3,4)$.

There is currently much interest in finding biomarkers that allow early detection of microvascular damage and predict the course of SSc, and, although it is still a matter of debate, various molecules have been evaluated (5-7). Among them, klotho has captured the attention of researchers because of its central role in mineralisation, electrolytic balance, insulin sensitivity, cell renewal and reparative processes. Some studies have found that SSc patients have
Corresponding author: Rossella Talotta Department of Rheumatology, University Hospital ASST-FBF-Sacco Via GB Grassi, 74 - 20157 Milano, Italy E-mail: talotta1@virgilio.it 
lower serum klotho concentrations than healthy controls, albeit without any association with clinical manifestations (8-10). However, no previous study has attempted to evaluate the association between serum klotho levels and nailfold capillaroscopic images in SSc patients.

\section{MATERIALS AND METHODS}

\section{Patients}

We retrospectively evaluated 54 consecutive patients diagnosed as having $\mathrm{SSc}$ on the basis of the 2013 EULAR/ACR criteria (4). They were randomly selected from a previous study designed to evaluate serum klotho concentrations in SSc patients (local Ethics Committee No. 88 of 5 February 2015) (10). All of the patients gave their informed consent to the study analyses and procedures.

A record was made of their demographic and clinical characteristics, including disease severity assessed using the Medsger's scale (11) and skin fibrosis assessed using the modified Rodnan skin score (mRSS), pulmonary and cardiac involvement [forced vital capacity (FVC), diffusing capacity/transfer factor of the lung for carbon monoxide per unit of alveolar volume (DLCO/AV), high-resolution computed chest tomography (HRCT), and 2D echocardiography (2D-ECHO)], the results of laboratory tests [serum creatinine level, erythrocyte sedimentation rate (ESR), Creactive protein (CRP), and autoantibodies], and concomitant treatments.

All of the patients underwent a nailfold capillaroscopic examination, and the acquired images were reviewed by two independent investigators. Capillaroscopic patterns were considered normal or not according to the size, distribution, number and shape of the capillaries (12). Scleroderma patterns were scored on the basis of the classification by Cutolo et al. (13). Briefly, after applying a drop of cedar oil on the cuticles of the $2^{\text {nd }}$, $3^{\text {rd }}, 4^{\text {th }}$ and $5^{\text {th }}$ fingers, nailfold capillaroscopy was performed using an optical microscope with a magnification lens of $200 x$ to analyse four consecutive areas of $1 \mathrm{~mm}$ (14), and the presence of avascular areas (the lack of capillaries in an area of $500 \mu \mathrm{m}$ ) or decreased capillary density, megacapillaries (giant loops with a diameter $>50 \mu \mathrm{m}$ ), and neo-angiogenesis (arborescent capillaries) was recorded. A numeric score was arbitrarily assigned to capillaroscopic patterns $(0=$ normal; $1=$ early scleroderma pattern; 2 $=$ active scleroderma pattern; 3 = late scleroderma pattern).

\section{Laboratory analyses}

Venous blood samples were collected from each patient, centrifuged, and the serum concentration of soluble klotho was measured by means of an enzyme-linked immunosorbent assay (ELISA) with a sensitivity of $0.1 \mathrm{ng} / \mathrm{mL}$ using a monoclonal anti-KL antibody and a KL-HRP conjugate (My Biosource, San Diego, CA, USA).

\section{Statistical analysis}

The demographic, clinical and pharmacological data were descriptively analysed using SPSS statistical software, version 23, and are reported as mean values \pm standard deviation (SD) or median values, interquartile (IQ) and range depending on their Gaussian or non-Gaussian distribution. The associations between serum klotho concentrations and other data were evaluated by means of regression analysis [analysis of variance (ANOVA)] with the significance level set at $\mathrm{p}<0.05$. Pearson's chi-squared test was used to analyse the associations between the nominal variables.

\section{RESULTS}

\section{Patient characteristics}

Fifty-four SSc patients [47 females (87\%); median age 68.0 years (IQ 18, range 30-79); median disease duration 11.0 years (IQ 7, range 4-38)], who were diagnosed on the basis of the 2013 EULAR/ACR criteria, were consecutively enrolled between October 2014 and April 2016. Forty-three (79.6\%) had a limited form of the disease and 11 (20.4\%) a diffuse form. The mean Medsger's scale score of severity was $4.0 \pm 2.2$, and the median modified Rodnan skin score (mRSS) was 4.0 (IQ 5, range 0-23).

Antinuclear antibodies (ANAs) were detected in 53 patients $(98.1 \%)$, anti-extract- 
able nuclear antigen (ENA) antibodies in 30 (55.6\%), anti-centromere antibodies (ACAs) in 27 (50\%), anti-SCL70 antibodies in $13(24.1 \%)$, and anticardiolipin antibodies (ACLAs) in three (5.6\%). Two-D echocardiography showed signs of PAH in seven patients (13\%), and lung HRTC detected pulmonary fibrosis in $22(40.7 \%)$. Nine patients $(16.7 \%)$ had DUs, seven $(13 \%)$ had calcinosis. The median ESR was $19.5 \mathrm{~mm}$ at the first hour (IQ 31; range 2-58), the median CRP level was $2.25 \mathrm{mg} / \mathrm{L}$ (IQ 4; range $1-24)$, and the mean serum creatinine level was $0.82 \pm 0.22 \mathrm{mg} / \mathrm{dL}$. Pulmonary function tests showed that mean VC was $99.8 \pm 15.5 \%$ and mean DLCO/AV 81.0 $\pm 14.2 \%$.

Nailfold capillaroscopy revealed a normal pattern in 14 patients (25.9\%), an early SSc pattern in eight (14.8\%), an active SSc pattern in $21(38.9 \%)$, and a late SSc pattern in $11(20.4 \%)$. Twenty-one patients (38.9\%) had avascular areas, $35(64.8 \%)$ had at least one megacapillary, and 16 (29.6\%) showed neo-angiogenesis.

Sixteen patients $(29.6 \%)$ were taking prednisone (2.5-12.5 mg/day), 18 (33.3\%) were taking calcium channel blockers, and 15 $(27.8 \%)$ were taking hydroxychloroquine (200-400 mg/day). All of the patients were receiving periodic intravenous infusions of prostanoids (alprostadil in 29, and iloprost in 25).

Table I resumes the demographic and clinical characteristics of the patients.

\section{Klotho concentrations}

The median serum klotho concentration was $0.29 \mathrm{ng} / \mathrm{mL}$ (IQ 1, range $0-2$ ): the patients with a normal nailfold capillaroscopic pattern had a median concentration of $0.89 \mathrm{ng} / \mathrm{mL}$ (IQ 1, range $0-2$ ); those with an early SSc pattern had a median concentration of $0.25 \mathrm{ng} / \mathrm{mL}$ (IQ 1, range $0-1)$; those with an active SSc pattern had a median concentration of $0.25 \mathrm{ng} / \mathrm{mL}$ (IQ 1 , range 0-1); and those with a late SSc pattern had a median concentration of $0.25 \mathrm{ng} /$ $\mathrm{mL}$ (IQ 1, range 0-1).

Regression analysis (ANOVA) showed a significant inverse correlation between serum klotho concentrations and the severity of the capillaroscopic pattern $(\mathrm{p}=0.02$;
Table I - Characteristics of the studied patients.

\begin{tabular}{|l|c|}
\hline No. of patients & 54 \\
\hline Females/males & $47 / 7$ \\
\hline Median age, years (IQ, range) & $68.0(18,30-79)$ \\
\hline Limited/diffuse disease & $43 / 11$ \\
\hline Median disease duration, years (IQ, range) & $11.0(7,4-38)$ \\
\hline Median Medsger scale score ( \pm SD) & $4.0 \pm 2.2$ \\
\hline Median mRSS (IQ, range) & $4.0(5,0-23)$ \\
\hline ANA positivity, No. (\%) & $53(98.1 \%)$ \\
\hline ACA positivity, No. (\%) & $27(50 \%)$ \\
\hline Anti-scl-70 antibody positivity, No. (\%) & $13(24.1 \%)$ \\
\hline Digital ulcers, No. (\%) & $9(16.7 \%)$ \\
\hline Interstitial lung disease, No. (\%) & $22(40.7 \%)$ \\
\hline Pulmonary artery hypertension, No. (\%) & $7(13 \%)$ \\
\hline Normal/abnormal nailfold capillaroscopic findings & $14 / 40$ \\
\hline
\end{tabular}

$I Q$, interquartile; SD, standard deviation; mRSS, modified Rodnan skin score; ANA, anti-nuclear antibody; ACA, anti-centromere antibody.

t -2.2284; Figure 1), which was not influenced by concomitant treatment. However, they did not significantly correlate with the presence of avascular areas, megacapillaries or neo-angiogenesis when each of these variables was individually evaluated. Logistic regression did not reveal any significant association between the risk of developing digital ulcers and the nailfold capillaroscopic pattern, serum klotho levels or concomitant medications. Pearson's chi-squared test showed a significant asso-

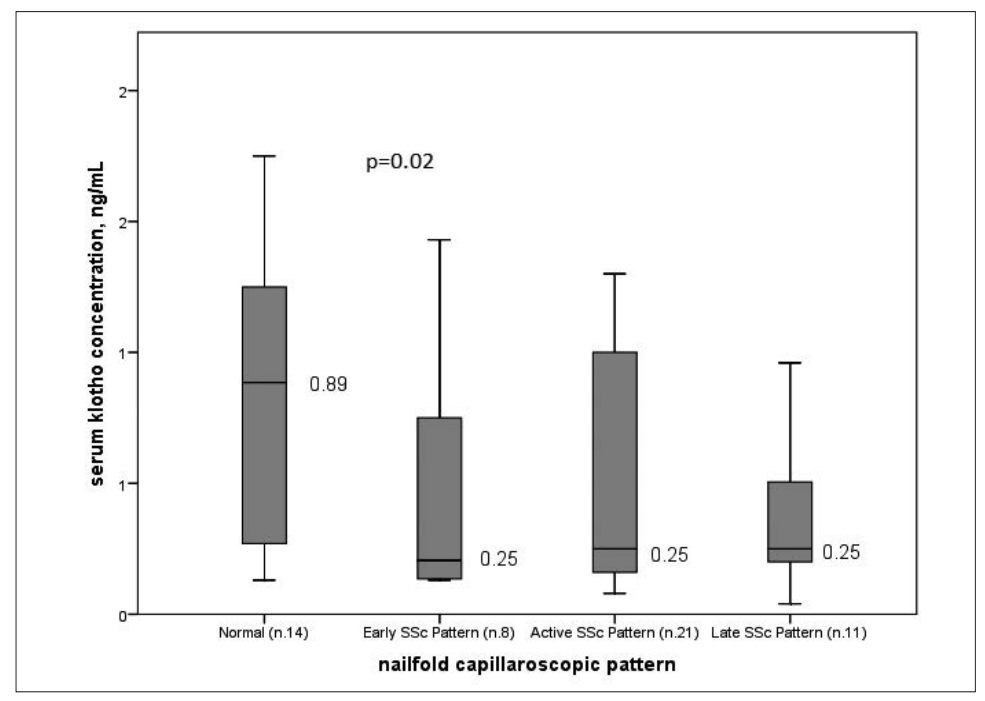

Figure 1 - Serum klotho concentrations in SSc patients with different nailfold capillaroscopic patterns. 
ciation between avascular areas detected at nailfold capillaroscopy and the presence of calcinosis $(p=0.006)$, which is in line with the results of previous studies (15). Calcinosis was significantly associated with ACA positivity $(\mathrm{p}=0.04)$ and the mRSS $(p=0.02)$, although these variables did not correlate with the capillaroscopic findings.

\section{DISCUSSION}

The pathogenesis of SSc is characterised by a complex network of vascular cells, fibroblasts and cells belonging to the immune system. Endothelial dysfunction and fibrosis are both hallmarks of the disease, thus making it unique in the spectrum of connective tissue diseases; however, reduced perfusion related to an imbalance between the factors governing vasoconstriction and vasodilatation, as well as to anatomical modifications of microvessels, are ultimately responsible for Raynaud's phenomenon, DUs, teleangiectasias, PAH and calcinosis, which reflect the global involvement of the microcirculation in different systems (16).

Nailfold capillaroscopy is a non-invasive and reliable means of assessing the morphological characteristics of nailfold capillaries, which are part of the terminal circulation and may undergo particular changes during vasculopathic processes such as those developing in SSc patients. It is therefore useful for detecting early anatomical details suggesting SSc, distinguishing early SSc from non-pathological Raynaud's phenomenon (17), and helping physicians to monitor and predict the course of the disease. Although there is still no good method of quantitative scoring, a number of studies have found a good correlation between worsening nailfold capillaroscopic patterns and visceral organ impairment or the risk of developing DUs in SSc patients (2). Finally, other studies have shown a significant association between specific capillaroscopic pictures or patterns and soluble biomarkers, including vascular endothelial growth factor (VEGF) and cluster of differentiation $40 \mathrm{~L}$ (CD40L) $(5,18)$.

Klotho is a single-pass transmembrane soluble protein governing vascular stability, hydro-electrolytic balance and tissue repair
(19). It has two isoforms (alpha and beta), with alpha-klotho being expressed throughout the arterial tree and involved in the development of vascular ectopic calcifications $(20,21)$. Reduced serum klotho concentrations have been observed in the elderly and during end-stage kidney disease, and it has been shown that klotho may prevent vasoconstriction, endothelial dysfunction and oxidative stress by regulating $\mathrm{Ca} 2+$ ion influx into microvascular cells, as well as the concentrations of reactive oxygen species (ROS) and nitric oxide (NO) $(22,23)$. We and other authors have previously found a deficit in serum soluble klotho in SSc patients $(8,10)$, and Mazzotta et al. have recently demonstrated that the addition of soluble alpha-klotho to human microvascular endothelial cells in vitro has pro-angiogenetic effects that allow wound healing (24). This is the first study to investigate the association between serum klotho levels and nailfold capillaroscopic patterns in SSc patients. We found that low levels were related to worse capillaroscopic patterns as classified by Cutolo et al., although there were no significant associations when avascular areas, megacapillaries and neo-angiogenesis were considered individually. Our findings also confirmed that a late scleroderma pattern was associated with calcinosis, ACA positivity, and worse mRSS values.

The main limitations of this study are that no healthy subjects or those suffering from primitive Raynaud's phenomenon were enrolled as control groups, that only the total concentration of klotho was evaluated (without distinguishing between alpha- and betaklotho or among the soluble, secreted and transmembrane isoforms), and that the nailfold capillaroscopic images were assessed qualitatively rather than quantitatively. Moreover, the standardised range of serum klotho concentration is still arbitrary as different methodologies have been used in previous studies of patients and healthy subjects.

\section{CONCLUSIONS}

On the basis of our findings, serum soluble klotho might be considered a potential biomarker of microvascular dysfunction 
in SSc as low levels significantly correlate with worse capillaroscopic patterns. Future studies are needed in order to ascertain the usefulness of soluble klotho in predicting the risk of developing DUs or PAH, both of which are due to irreversible changes in the microvascular tree, or monitoring the efficacy of vasodilating therapies.

Acknowledgements: the authors would like to thank the medical and paramedical staff who contributed to collecting the data and serum samples.

Contributions: RT conceived the idea, wrote the protocol, enrolled the patients, collected and statistically analysed the data, reviewed the capillaroscopic images, and wrote the final manuscript; FR, MCD, MA collected the clinical data; SB collected and stored the serum samples; TL and TV made the laboratory analyses; SS and MCD performed the nailfold capillaroscopy and reviewed the images; FA and PS critically reviewed the paper. All of the authors approved the final version of the manuscript.

\section{REFERENCES}

1. Yamamoto T, Katayama I. Vascular changes in bleomycin-induced scleroderma. Int J Rheumatol. 2011; 2011: 270938.

2. Smith V, Thevissen K, Trombetta AC, et al.; EULAR Study Group on Microcirculation in Rheumatic Diseases. Nailfold capillaroscopy and clinical applications in systemic sclerosis. Microcirculation. 2016; 23: 364-72.

3. LeRoy EC, Medsger TA Jr. Criteria for the classification of early systemic sclerosis. J Rheumatol. 2001; 28: 1573-6.

4. van den Hoogen F, Khanna D, Fransen J, et al. 2013 classification criteria for systemic sclerosis: an American college of rheumatology/European league against rheumatism collaborative initiative. Ann Rheum Dis. 2013; 72: 1747-55.

5. Yalçınkaya Y, Adın-Çınar S, Artim-Esen B, et al. Capillaroscopic findings and vascular biomarkers in systemic sclerosis: Association of low CD40L levels with late scleroderma pattern. Microvasc Res. 2016; 108: 17-21.

6. Yalçinkaya Y, Çinar S, Artim-Esen B, et al. The relationship between vascular biomarkers and disease characteristics in systemic sclerosis: elevated MCP-1 is predominantly associated with fibrotic manifestations. Clin Exp Rheumatol. 2016; 34: 110-4.

7. Chora I, Guiducci S, Manetti M, et al. Vascular biomarkers and correlation with peripheral vasculopathy in systemic sclerosis. Autoimmun Rev. 2015; 14: 314-22.
8. Ahmadi R, Hajialilo M, Ghorbanihaghjo A, et al. FGF-23, Klotho and vitamin D levels in scleroderma. Iran J Public Health. 2017; 46: 530-6.

9. Hajialilo M, Noorabadi P, Tahsini Tekantapeh S, Malek Mahdavi A. Endothelin-1, $\alpha$-Klotho, $25(\mathrm{OH})$ Vit D levels and severity of disease in scleroderma patients. Rheumatol Int. 2017 [Epub ahead of print].

10. Talotta R, Bongiovanni S, Letizia T, et al. Measurement of serum klotho in systemic sclerosis. Dis Markers. 2017; 2017: 9545930.

11. Geirsson AJ, Wollheim FA, Akesson A. Disease severity of 100 patients with systemic sclerosis over a period of 14 years: using a modified Medsger scale. Ann Rheum Dis. 2001; 60: 1117-22.

12. Andrade LE, Gabriel Júnior A, Assad RL, et al. Panoramic nailfold capillaroscopy: a new reading method and normal range. Semin Arthritis Rheum. 1990; 20: 21-31.

13. Cutolo M, Sulli A, Pizzorni C, et al. Nailfold videocapillaroscopy assessment of microvascular damage in systemic sclerosis. J Rheumatol. 2000; 27: $155-60$

14. Ruaro B, Sulli A, Smith V, et al. Microvascular damage evaluation in systemic sclerosis: the role of nailfold videocapillaroscopy and laser techniques. Reumatismo. 2017; 69: 147-55.

15. Morardet L, Avouac J, Sammour M, et al. Late nailfold videocapillaroscopy pattern associated with hand calcinosis and acro-osteolysis in systemic sclerosis. Arthritis Care Res (Hoboken). 2016; 68: 366-73.

16. Prete M, Fatone MC, Favoino E, Perosa F. Raynaud's phenomenon: from molecular pathogenesis to therapy. Autoimmun Rev. 2014; 13: 655-67.

17. Cutolo M, Sulli A, Smith V. How to perform and interpret capillaroscopy. Best Pract Res Clin Rheumatol. 2013; 27: 237-48.

18. De Santis M, Ceribelli A, Cavaciocchi F, et al. Nailfold videocapillaroscopy and serum VEGF levels in scleroderma are associated with internal organ involvement. Auto Immun Highlights. 2016; 7: 5.

19. Bian A, Neyra JA, Zhan M, Hu MC. Klotho, stem cells, and aging. Clin Interv Aging. 2015; 10: 1233-43.

20. Lim K, Groen A, Molostvov G, et al. $\alpha$-Klotho Expression in Human Tissues. J Clin Endocrinol Metab. 2015; 100: E1308-18.

21. Kurosu H, Kuro-O M. The Klotho gene family as a regulator of endocrine fibroblast growth factors. Mol Cell Endocrinol. 2009; 299: 72-8.

22. Kusaba T, Okigaki M, Matui A, et al. Klotho is associated with VEGF receptor-2 and the transient receptor potential canonical-1 $\mathrm{Ca} 2+$ channel to maintain endothelial integrity. Proc Natl Acad Sci U S A. 2010; 107: 19308-13.

23. Six I, Okazaki H, Gross P, et al. Direct, acute effects of Klotho and FGF23 on vascular smooth muscle and endothelium. PLoS One. 2014; 9: e93423.

24. Mazzotta C, Manetti M, Rosa I, et al. Proangiogenic effects of soluble $\alpha$-Klotho on systemic sclerosis dermal microvascular endothelial cells. Arthritis Res Ther. 2017; 19: 27. 